\title{
FIRST ORDER, QUASI-STATIC, SOI CHARGE CONSERVING POWER DISSIPATION MODEL
}

\author{
Sameer Sharma and L G. Johnson \\ Oklahoma State University, Stillwater, OK 74075
}

\begin{abstract}
Conventional MOS models for circuit simulation assume that the channel capacitances do not contribute to net power dissipation. Numerical integration of channel currents and instantaneous terminal voltages however shows the existence of higher order dissipating terms. To overcome these limitations, we present a self-consistent, first order, quasi-static power dissipation model that is able to predict dissipative (transport) and conserved (charging) current components. Charge conservation is insured by using the current continuity equation. An analytical expression for energy stored in the channel is derived by separating out current components that contribute to net power dissipation. The power dissipation estimation is made computationally efficient by leaving out energy conserving terms.
\end{abstract}

\section{INTRODUCTION}

Modeling is a process of accurately representing the behavior of a device to be used in a circuit simulator. Designers need these reliable and accurate device models for circuit development. With the growth of CMOS technology, MOSFET modeling has become increasingly important. The accurate modeling of the MOSFET channel capacitance has been an ongoing effort for many decades. First, Meyer's [1] reciprocal capacitive model, then Ward's [2] charge-based non-reciprocal capacitance model have been used. Many papers have been written on the comparison of these models. Some [3-5] claim that Meyer's model fails due to charge non-conservation which justifies the usage of charge-based models, while others claim [6-7] that the charge non-conservation is mainly due to the incorrect mathematical modeling of non-linear capacitance by the simulation software. Recent papers on fielddependent mobility [8] and laterally asymmetrical doping [9] have now shown inconsistencies in Ward's model, which artificially partitions the channel charge into source and drain components. As pointed out by Fossum [10], it is not clear whether we have explored all other possibilities; we may be able to achieve a better result with a different channel partition or may be with no partition at all.

Many models have also been put forward to analyze the charging and the trans-capacitive current components. One of such models by Lim and Fossum [11] has a first order transient transport current and suggests the difference between non-reciprocal capacitive elements to be responsible for this current. We show that this model is correct for transistor current computation; however it is inconsistent and has some drawbacks when used to predict power consumption. These drawbacks are:

- Lim-Fossum's equations use Ward's channel charge partition model. 
- The MOS capacitors dissipate power and the trans-capacitive term used in the charge model includes both dissipating as well as conserved components which are not separated.

The charge partition model puts a constraint only on charge conservation. Even though the model predicts the channel charge correctly to first order, the device power is only predicted to zero order. The model may not include complete first order trans-capacitive currents due to the redistribution of the charges in the channel. This could cause the actual output waveform and delay to deviate from the simulation results [12]. In reality, the MOS channel is not purely an energy storage device [13]. Thus, it is not appropriate to leave out higher order dissipating terms due to charge redistribution as they become significant at higher frequencies.

Though many papers/chapters [3-5, 14-17] have been written on the transient transport current, no one has found a solution to separate the transport and charging current components. This makes our model and the closed form expressions for the dissipative and conserved currents significant. We have developed a self consistent, quasi-static, charge conserving, first order power dissipation model. It analyzes the first order power dissipation and computes the energy function for the conserved component of the charge storage. The existence of the energy function makes it possible to exclude energy conserving terms that do not contribute to power dissipation, making the total power estimation computationally efficient.

The rest of the paper is organized as follows. In the second section, we have used a one dimensional MOSFET transistor model with the current continuity equation to compute the channel currents and the channel charges as well as the currents at the source and the drain ends. In the third section, we have computed the power by integrating the power density over the entire channel. This leads to the derivation of an energy function in section four. In section five, we discuss the first order dynamic power dissipation model. Using the conserved power components, we have separated the conserved and dissipative current components in section six. Finally, we have developed an equivalent circuit by following the method used by LimFossum and verified the results for current and charge. Even though they used a charge partition instead of solving exactly as we have, both models predict the same source and drain currents, and hence the same terminal capacitances. However, we are able to separate out these capacitances into conserved and dissipative components.

\section{CHARGE DISTRIBUTION CALCULATION}

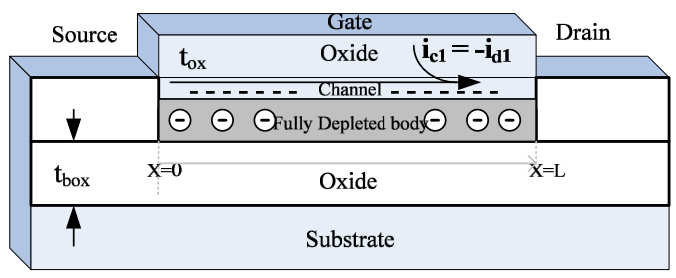

Fig. 1. SOI MOSFET Structure

In order to obtain an analytical solution, the current flow is considered in one dimension parallel to the surface of the device. It is assumed that the region under the channel is completely depleted of mobile charges. This fully depleted assumption for SOI MOSFET's 
helps us to make use of a linear relationship between the body and the surface potential to compute the stored energy function without partitioning the channel charge. The linear relation also provides a simplified charge model and terminal currents. It should be noted that solving these equations involves complicated algebraic calculations that are practically impossible without modern mathematics tools like "Mathematica" [18].

Fig. 1 shows NMOS SOI transistor. The charge per unit length $\left(q_{c}\right)$ at a position $\mathrm{x}$ along the channel is given by

$$
q_{c}(x)=-c_{o x}\left(v_{g b}-v_{f b}-v_{c b}(x)-\phi+q_{b}(x) / c_{o x}\right)
$$

Similarly, the body charge (back gate) per unit length $\left(q_{b}\right)$ at $\mathrm{x}$ can be written as

$$
q_{b}(x)=-c_{o x}\left(k_{1}+\alpha v_{c b}(x)\right)
$$

where $v_{f b}, v_{g b}$ and $v_{c b}$ are the flat band, gate and channel voltages with respect to the body. $k_{1}$ and $\alpha$ are body effect coefficients. $c_{o x}=W\left(c_{o x} / A\right)$ is the oxide capacitance per unit length, where $\mathrm{W}$ is the channel width. Charge conservation is insured by defining the gate charge per unit length $q_{g}$ as

$$
q_{g}=-\left(q_{b}+q_{c}\right)
$$

It will be convenient to define the channel charge per unit length at the source $(x=0) q_{S}$ and the drain $(x=L) q_{d}$ and their time derivatives as

$$
q_{s}=-c{ }^{v}{ }^{v_{g s t}}
$$

where $v_{g s t}=v_{g b}-v_{t}-v_{s b}$

$$
\frac{d}{d t} q_{s}=-c_{o x} \frac{d}{d t} v_{g s t}
$$

In equation (4), $v_{t}$ is the threshold voltage. The body effect requires including the dependence of the threshold voltage on source terminal voltage and the substrate charge parameter [22].

$$
v_{t}\left(v_{s b}\right)=v_{t 0}+\alpha v_{s b}
$$

where $v_{t 0}=v_{f b}+k_{1}+\phi$ is the threshold voltage at zero $v_{s b}$, and $\phi$ is the fermi potential. At the drain end,

$$
\begin{aligned}
& q_{d}=-c_{o x} v_{g d t} \\
& \text { where } v_{g d t}=v_{g b}-v_{t}-v_{s b}-(1+\alpha)\left(v_{d b}-v_{s b}\right) \\
& \frac{d}{d t} q_{d}=-c_{o x} \frac{d}{d t} v_{g d t}
\end{aligned}
$$

It is assumed that positive current flows into the drain and velocity saturation effects can be neglected. Assuming strong inversion, diffusion current in the channel is small. Drift current at a distance $\mathrm{x}$ along the channel can be written as

$i_{c}(x, t)=q_{c}(x, t) \mu \frac{d}{d x} v_{c b}(x)$

where $\mu$ is the charge carrier mobility in the channel. Charge conservation is assured using 
the one dimensional continuity equation

$$
\frac{d}{d x} i_{c}(x, t)=-\frac{d}{d t} q_{c}(x, t)
$$

Using (9) in (10) gives

$$
\frac{d}{d x}\left[q_{c}(x, t) \mu \frac{d}{d x} v_{c b}(x)\right]=-\frac{d}{d t} q_{c}(x, t)
$$

Taking the spatial derivatives of charge per unit length as a linear function of potential along the channel as in equation (1) and (2) gives

$$
\frac{d}{d x} q_{c}(x, t)=(1+\alpha) c_{o x} \frac{d}{d x} v_{c b}(x)
$$

Substituting $\frac{d}{d x} v c b(x)$ in (11) and rearranging terms gives

$$
\frac{d}{d x}\left[q_{c}(x, t) \frac{d}{d x} q_{c}(x, t)\right]=-\frac{(1+\alpha)}{\mu} c_{o x} \frac{d}{d t} q_{c}(x, t)
$$

In the quasi-static approximation, equation (13) can be solved iteratively to compute the current and the charge in the channel by expanding $q_{c}=q_{c 0}+q_{c 1}+\ldots$. where $q_{c 0}$ is a function of terminal voltages only and $q_{c 1}$ is a linear function of first order time derivatives of terminal voltages. In terms of the steady state (zero order) charge per unit length at any position $\mathrm{x}$ along the channel, equation (13) reduces to

$$
\frac{d}{d x}\left(q_{c 0} \frac{d}{d x} q_{c 0}\right)=0
$$

Performing integration from the source $(x=0)$ to the drain $(x=L)$, the zero order charge along the channel becomes

$$
q_{c 0}=-\sqrt{\left(q_{s}^{2}(1-x / L)+q_{d}^{2} x / L\right.}
$$

and the steady state drift current component of equation (9) simplifies to

$$
I_{c 0}=\frac{\mu}{(1+\alpha) c_{o x}} q_{c 0} \frac{d}{d x} q_{c 0}
$$

Equation (16) gives the usual equation for steady current neglecting velocity saturation, which is shown in Table I. The first order current and charge can be found by keeping terms of first order in time derivatives in equation (13)

$$
\frac{d}{d x}\left(q_{c 0} \frac{d}{d x} q_{c 1}+q_{c 1} \frac{d}{d x} q_{c 0}\right)=-\frac{(1+\alpha)}{\mu} c_{o x} \frac{d}{d t} q_{c 0}
$$

Rearranging the terms, equation for the first order channel charge simplifies to

$$
\left.q_{c 1}=-\frac{(1+\alpha)}{\mu} c_{o x} \frac{1}{q_{c 0}} \frac{d}{d t} \int\left(\int_{c 0}[x] d x\right) d x\right)
$$

and the first order current reduces to

$$
i_{c 1}=\frac{\mu}{(1+\alpha) c_{o x}}\left(q_{c 0} \frac{d}{d x} q_{c 1}+q_{c 1} \frac{d}{d x} q_{c 0}\right)
$$

Finally, equation (19) can be solved to compute the first order channel current at the source $i_{s 1}=i_{c 1}(x=0)$ and the drain $i_{d 1}=-i_{c 1}(x=L)$ ends in all regions of operation. We have assumed pinch-off saturation which occurs when $q_{d}=0$ for $v_{d s} \geq \frac{\left(v_{g s}-v_{t}\right)}{(1+\alpha)}$. In cut-off, it is 
assumed that both $q_{d}=0$ and $q_{s}=0$. Table 1 summarizes the charge and current in all regions of operations.

\subsection{Derivation of First Order Channel Charge and Channel Current}

The equations for first order channel charge and first order currents were used in the previous section, however, the derivations were not shown, which is given in this section. These derivations are one of the most important findings of our research.

The first order channel charge allows calculating the first order channel current without the charge partition, which can be used to calculate the first order drain and source currents. The first order channel current also makes it possible to derive the conserved and dissipative power components.

Taking charge density as a function of potential along the channel, and keeping terms of first order in time derivatives, current continuity equation (17) can be rearranged to be written in terms of first order channel charge per unit length as

$$
q_{c 1}=-\frac{c_{O x}(1+\alpha)}{\mu} \frac{1}{q_{c 0}} \frac{d}{d t} \iint q_{c 0}[x] d x d x+c 1 x+c 0
$$

where $q_{c 0}$ is the zero order channel charge density and is given by equation (15). $c 1$ and $c 0$ are constants of integration and can be calculated using the boundary condition $q_{c 1}=0$ at $x=0$ and $x=L$

$$
\begin{aligned}
c 0= & \left.\frac{c_{o x}(1+\alpha)}{\mu} \frac{d}{d t} \iint q_{c 0}[x] d x d x\right|_{x \rightarrow 0} \\
c 0= & \frac{4}{15} \frac{{ }^{c} o x(1+\alpha)}{\mu} L^{2} q_{s} \frac{\left(4 q_{d} q_{s} \frac{d}{d t} q_{d}-5 q_{d}^{2} \frac{d}{d t} q_{s}+q_{s}^{2} \frac{d}{d t} q_{s}\right)}{\left(-q_{d}{ }^{2}+q_{s}^{2}\right)^{3}} \\
c 1= & \left.\frac{c_{o x}(1+\alpha)}{\mu} \frac{d}{d t} \iint q_{c 0}[x] d x d x\right|_{x \rightarrow L} \\
c 1= & \frac{4}{15} \frac{c o x}{\mu}(1+\alpha) \\
& \frac{\left(-q_{d} \frac{d}{d t} q_{d}\left(q_{d}{ }^{5}-5 q_{d}^{3} q_{s}{ }^{2}+4 q_{s}{ }^{5}\right)-q_{s}\left(4 q_{d}{ }^{5}-5 q_{d}{ }^{2} q_{s}{ }^{3}+q_{s}{ }^{5}\right) \frac{d}{d t} q_{s}\right)}{\left(-q_{d}{ }^{2}+q_{s}{ }^{2}\right)^{3}}
\end{aligned}
$$

Substituting the values of $c 0, c 1$ and $q_{c 0}$ in (20), the first order charge at any point $\mathrm{x}$ along the channel then becomes 


$$
\begin{aligned}
& q_{c 1}=-\frac{1}{15 \sqrt{\frac{q_{s}^{2}(L-x)+q_{d}{ }^{2} x}{L}}}\left\{4 C _ { c } L \left(\frac{L q_{s}^{4}\left(4 q_{d} q_{s} \frac{d}{d t} q_{d}-5 q_{d}{ }^{2} \frac{d}{d t} q_{s}+q_{s}{ }^{2} \frac{d}{d t} q_{s}\right)}{\left(-q_{d}{ }^{2}+q_{s}{ }^{2}\right)^{3}}+\right.\right. \\
& \left(\left(q_{d}{ }^{5} \frac{d}{d t} q_{d}-q_{s}{ }^{5} \frac{d}{d t} q_{s}+4 q_{d}{ }^{3} q_{s}{ }^{2}\left(-\frac{d}{d t} q_{d}+\frac{d}{d t} q_{s}\right)+4 q_{d}{ }^{2} q_{s}^{3}\left(-\frac{d}{d t} q_{d}+\frac{d}{d t} q_{s}\right)-\right.\right. \\
& \left.\left.\left.q_{d^{q}}{ }^{4}\left(4 \frac{d}{d t} q_{d}+\frac{d}{d t} q_{s}\right)+q_{d}{ }^{4} q_{s}\left(\frac{d}{d t} q_{d}+4 \frac{d}{d t} q_{s}\right)\right) x\right) /\left(q_{d}-q_{s}\right)^{2}\left(q_{d}+q_{s}\right)^{3}\right)- \\
& \frac{1}{\left(q_{d}{ }^{2}-q_{s}{ }^{2}\right)^{3}}\left(\frac{q_{s}{ }^{2}(L-x)+q_{d}{ }^{2} x}{L}\right)^{3 / 2}\left(L q_{s}\left(4 q_{d} q_{s} \frac{d}{d t} q_{d}-5 q_{d}{ }^{2} \frac{d}{d t} q_{s}+q_{s}{ }^{2} \frac{d}{d t} q_{s}\right)-\right. \\
& \left.\left.\left.\left(q_{d}-q_{s}\right)\left(q_{d}+q_{s}\right)\left(q_{d} \frac{d}{d t} q_{d}-q_{s} \frac{d}{d t} q_{s}\right) x\right)\right)\right)
\end{aligned}
$$

The first order channel current at any position $\mathrm{x}$ along the channel can now be estimated using equation (19). Taking derivatives of $q_{c 0}$ and $q_{c l}$, and substituting the corresponding values, equation (19) expands to

$$
\begin{aligned}
& i_{c 1}=\frac{L}{15\left(q_{d}-q_{s}\right)^{2}\left(q_{d}+q_{s}\right)^{3}}\left\{4 \left(q_{d} \frac{d}{d t} q_{d}\left(q_{d}{ }^{4}+q_{d}{ }^{3} q_{s}-4 q_{d}{ }^{2} q_{s}{ }^{2}-4 q_{d} q_{s}{ }^{3}-4 q_{s}{ }^{4}\right)+\right.\right. \\
& \left.q_{d} \frac{d}{d t} q_{s}\left(4 q_{d}{ }^{4}+4 q_{d}{ }^{3} q_{s}+4 q_{d}{ }^{2} q_{s}{ }^{2}-q_{d} q_{s}{ }^{3}-q_{s}{ }^{4}\right)\right\}-10\left(q_{d}+q_{s}\right)\left(\sqrt{q_{s}{ }^{2}\left(1-\frac{x}{L}\right)+q_{d}} \frac{2}{L}\right. \\
& \left\{q_{s}\left(2 q_{d} \frac{d}{d t} q_{d} q_{s}-\left(3 q_{d}{ }^{2}-q_{s}{ }^{2}\right) \frac{d}{d t} q_{s}\right)-\left(q_{d}{ }^{2}-q_{s}{ }^{2}\right)\left(q_{d} \frac{d}{d t} q_{d}-q_{s} \frac{d}{d t} q_{s}\right) x\right\}
\end{aligned}
$$

As mentioned above, this is one of the most important findings of our research and can be solved to find the first order drain $\left(-i_{c 1}=i_{d 1}, x \rightarrow L\right)$ and source $\left(i_{c 1}=i_{s 1}, x \rightarrow 0\right)$ current components, which are shown in Table 1. These results obtained without partitioning the channel charge are in agreement with previous results by Lim and Fossum, which were obtained using Ward's partition. Therefore we have verified that Ward's partition is correct when the body charge has a linearly dependence on channel potential.

\section{CALCULATION OF MOSFET POWER}

This section describes the detailed derivations of MOS power components. To not to confuse with the general definition of the static and dynamic power terms, channel power components are defined as the zero and the first order powers. It should be pointed out that the zero order power is different than the static power. In general, static power is defined as being independent of time (time invariant). However, the zero order power that has been used in this study is time variant. Although there is no explicit dependence, it depends on the terminal voltages that change in time. In fact it includes exactly the dynamic powers terms that are proportional or are the functions of the terminal voltages, and ignore the explicit terms proportional to $\mathrm{dv} / \mathrm{dt}$. The first order power on the other hand, depends on the time derivatives of the terminal voltages, while the dynamic power that has been used in the literature depends on energy stored in external capacitances which includes zero order and some of the first order power dissipated in the transistor. 
The steady state current is usually used to determine power dissipation for MOS transistors. Charge redistribution in the channel causes additional power dissipation. In the quasi-static model, charge redistribution is assumed to happen instantaneously with no propagation delays. However, the channel charge density still changes as an indirect function of time through the dependence on time varying terminal voltages. This allows the use of the quasi-static model to predict the charge redistribution and the associated power dissipation.

The conventional charge model is based on the assumption that the MOSFET capacitors do not contribute any net power dissipation in the channel. But, the channel capacitances are not energy conserving [13]. They do have some higher order power dissipative terms due to the charge redistribution in the channel. These dissipative terms become significant at higher frequencies, which make it essential to include their effects for accurate power dissipation prediction.

Fig. 2 shows a MOS device. Considering a slice of thickness $\Delta x$, MOS channel can be thought of having two power components:

- Dissipative component (Fig. 2a): The current $i(x)$ flowing through the slice of thickness

$\Delta x$ having a potential $\Delta v$ which looks like a series resistance and results in the power dissipation of $i \Delta v$.

- Conserved component (Fig. 2b): The rate of change of charge that is building in the slice due to the difference in current $\Delta i$. This power change $v \Delta i$ is the energy stored in the charge at the potential $v(x)$

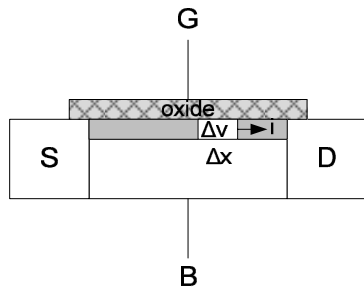

(a)

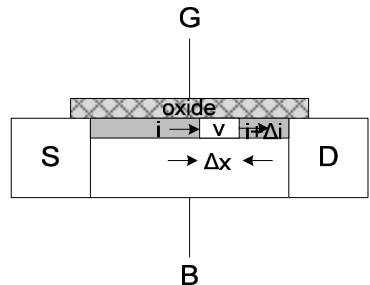

(b)

Fig. 2: MOS Channel Power Calculation

The instantaneous total power going into the transistor channel $P_{c}$ can be estimated by integrating the power density over the channel length:

$$
\begin{aligned}
P_{c} & =\int_{0}^{L} \frac{d}{d x}\left(i_{c}(x) v_{c b}(x)\right) d x \\
& =\int_{0}^{L} v_{c b}(x)\left(\frac{d}{d x} i(x)\right) d x+\int_{0}^{L} i_{c}(x)\left(\frac{d}{d x} v_{c b}(x)\right) d x
\end{aligned}
$$

where the first integral represents change in stored energy and second term represents power dissipation. Keeping non-zero terms to first order in time derivatives, equation (20) can be expanded as:

$$
P_{c}=P_{c 0}+P_{c 1, \text { diss }}+P_{c 1, \text { cons }}
$$


where

$$
\begin{aligned}
& P_{c 0}=\int_{0}^{L} I_{c 0}\left(\frac{d}{d x} v_{c b 0}(x)\right) d x=I_{c 0}\left(v_{d b}-v_{s b}\right) \\
& P_{c 1, \text { diss }}=\int_{0}^{L} i_{c 1}\left(\frac{d}{d x} v_{c b 0}(x)\right) d x \\
& P_{c 1, \text { cons }}=\int_{0}^{L} v_{c b 0}\left(\frac{d}{d x} i\right) d x
\end{aligned}
$$

The total instantaneous power $P$ into the transistor is the sum of channel power $P_{c}$ and gate power $P_{g 1, \text { cons }}$$$
P=P_{c}+P_{g 1, \text { cons }}
$$

where the gate power is

$$
P_{g 1, \text { cons }}=i_{g 1} v_{g b}
$$

Equation (25) represents the zero order power dissipation. Equation (26) represents the first order power dissipation due to the trans-capacitive transient current components and equation (27) represents the first order conserved power in the channel. Table 2 summarizes the power components.

\section{ENERGY STORED IN THE CHANNEL}

For the stored energy derivation, we have assumed that there is no charge leakage from the gate to the channel. However, energy is still supplied from the gate to drive the channel charges. It becomes necessary to add the conserved power contribution from the gate together with the channel conserved power. It is then possible to derive a closed form analytical solution for an energy function from the total conserved power.

\subsection{Energy function validation for the channel}

Clairaut's theorem states that, "If two second order partials are continuous, they will be equal". The same theorem can be used to check the equality of second order partial and verify the existence of the energy function.

Using equation (29), the conserved gate power can be written in terms of energy as

$$
P_{g 1, \text { cons }}=\frac{\partial E}{\partial v} \frac{d v}{d t}=\frac{\partial E_{g}}{\partial v_{g b}} \frac{d v_{g b}}{d t}+\frac{\partial E_{g}}{\partial v_{d b}} \frac{d v_{d b}}{d t}+\frac{\partial E_{g}}{\partial v_{s b}} \frac{d v_{s b}}{d t}
$$

where $E_{g}$ is the gate energy. Comparing (29) and (30), $\frac{\partial E}{\partial v}$ 's can be derived from the coefficients of $\frac{d v}{d t}$ as 


$$
\begin{aligned}
& \frac{\partial E_{g}}{\partial v_{s b}}=\frac{-2 c_{o x} L v_{g b t} v_{g s t}\left(2 v_{g d t}+v_{g s t}\right)}{3\left(v_{g d t}+v_{g s t}\right)^{2}} \\
& \frac{\partial E_{g}}{\partial v_{d b}}=\frac{-2 c_{o x} L v_{g b t} v_{g d t}\left(v_{g d t}+2 v_{g s t}\right)}{3\left(v_{g d t}+v_{g s t}\right)^{2}} \\
& \frac{\partial E_{g}}{\partial v_{g b}}=\frac{1}{6} c_{o x} L v_{g b t}\left(6-\frac{2\left(v_{g d t}-v_{g s t}\right)^{2}}{K\left(v_{g d t}+v_{g s t}\right)^{2}}\right)
\end{aligned}
$$

As mentioned earlier, energy function exists if and only if the second order partials are equal. Taking partials and comparing equations (31-33), it is found that the second order partials are not equal. Hence, energy function does not exist for the gate.

\subsection{Energy function validation for the channel}

Taking similar approach, $\frac{\partial E}{\partial v}$ 's are calculated from the coefficients of $\frac{d v}{d t}$ in $P_{c 1, \text { cons }}$ in (27) as

$$
\begin{aligned}
& \frac{\partial E_{c}}{\partial v_{s b}}=\frac{-c_{o x} L v_{g s t}\left(3\left(v_{g d t}+v_{g s t}\right)^{2}-4 v_{g b t}\left(2 v_{g d t}+v_{g s t}\right)\right.}{6\left(v_{g d t}+v_{g s t}\right)^{2}} \\
& \frac{\partial E_{c}}{\partial v_{d b}}=\frac{-c_{o x} L v_{g d t}\left(3\left(v_{g d t}+v_{g s t}\right)^{2}-4 v_{g b t}\left(v_{g d t}+2 v_{g s t}\right)\right.}{6\left(v_{g d t}+v_{g s t}\right)^{2}} \\
& \frac{\partial E_{c}}{\partial v_{g b}}=\frac{c_{o x} L\left(3\left(v_{g d t}+v_{g s t}\right)^{3}-4 v_{g b t}\left(v_{g d t}{ }^{2}+4 v_{g d t} v_{g s t}+v_{g s t}\right)\right.}{6 K\left(v_{g d t}+v_{g s t}\right)^{2}}
\end{aligned}
$$

where $E_{c}$ is the channel energy. It can again be shown that the second order partials are not equal and the channel also has no energy function from all of its conserved components.

\subsection{Energy function validation for combination of the gate and the channel}

Combining the conserved gate and channel power components, the first order conserved power can be written as

$$
P_{\text {cons }}=\left(\frac{\partial E_{c}}{\partial v_{g b}}+\frac{\partial E_{g}}{\partial v_{g b}}\right) \frac{d v_{g b}}{d t}+\left(\frac{\partial E_{c}}{\partial v_{d b}}+\frac{\partial E_{g}}{\partial v_{d b}}\right) \frac{d v_{d b}}{d t}+\left(\frac{\partial E_{c}}{\partial v_{s b}}+\frac{\partial E_{g}}{\partial v_{s b}}\right) \frac{d v_{s b}}{d t}
$$


which can again be solved to get $\frac{\partial E}{\partial v}$ 's from the coefficients of $\frac{d v}{d t}$ as

$$
\begin{aligned}
\frac{\partial E}{\partial v_{s b}} & =\frac{-c_{o x} L v_{g s t}\left(3\left(v_{g d t}+v_{g s t}\right)^{2}-4 v_{g b t}\left(2 v_{g d t}+v_{g s t}\right)\right.}{6\left(v_{g d t}+v_{g s t}\right)^{2}} \\
& +\frac{-2 c_{o x} L v_{g b t} v_{g s t}\left(2 v_{g d t}+v_{g s t}\right)}{3\left(v_{g d t}+v_{g s t}\right)^{2}} \\
\frac{\partial E}{\partial v_{d b}} & =\frac{-c_{o x} L v_{g d t}\left(3\left(v_{g d t}+v_{g s t}\right)^{2}-4 v_{g b t}\left(v_{g d t}+2 v_{g s t}\right)\right.}{6\left(v_{g d t}+v_{g s t}\right)^{2}} \\
& +\frac{-2 c_{o x} L v_{g b t} v_{g d t}\left(v_{g d t}+2 v_{g s t}\right)}{3\left(v_{g d t}+v_{g s t}\right)^{2}} \\
\frac{\partial E}{\partial v_{g b}} & =\frac{1}{6} c_{o x}^{L v_{g b t}\left(6-\frac{2\left(v_{g d t}-v_{g s t}\right)^{2}}{(1+\alpha)\left(v_{g d t}+v_{g s t}\right)^{2}}\right)} \\
+ & \frac{c_{o x} L\left(3\left(v_{g d t}+v_{g s t}\right)^{3}-4 v_{g b t}\left(v_{g d t}+4 v_{g d t} v_{g s t}+v_{g s t}\right)\right.}{6(1+\alpha)\left(v_{g d t}+v_{g s t}\right)^{2}}
\end{aligned}
$$

In this case, second order partials are equal. It confirms that an energy function exists from all of the conserved components of the gate and the channel.

\subsection{Energy Function Equation}

The existence of an energy function was validated in previous section. In this section an energy function equation is derived solving the partial differentials using

$$
\frac{\partial E}{\partial v_{j b}}\left(v_{g b}, v_{s b}, v_{d b}\right)=\frac{\partial E_{c}}{\partial v_{j b}}+\frac{\partial E_{g}}{\partial v_{j b}} ; j=g, s, d
$$

This method however, is cumbersome as it involves lots of algebra. A simple solution is possible by separating the gate power into two components.

$$
P_{g 1, \text { cons }}=i_{g 1} v_{g b}=i_{g 1} v_{g b t 0}+i_{g 1} v_{t 0}
$$

where $v_{g b t 0}=v_{g b}-v_{t 0}$ and $i_{g 1} v_{t 0}$ is the threshold power. In terms of energy, the gate power becomes 


$$
P_{g 1, \text { cons }}=\left(\frac{d E_{g b t}}{d v_{g b}}+\frac{d E_{t 0}}{d v_{g b}}\right) \frac{d v_{g b}}{d t}+\left(\frac{d E_{g b t}}{d v_{d b}}+\frac{d E_{t 0}}{d v_{d b}}\right) \frac{d v_{d b}}{d t}+\left(\frac{d E_{g b t}}{d v_{s b}}+\frac{d E_{t 0}}{d v_{s b}}\right) \frac{d v_{s b}}{d t}
$$

where $E_{t 0}$ and is the threshold energy function and $E_{g b t}$ is the energy function from the remaining gate terms. Equation (41) now can be rewritten as

$$
\frac{\partial E}{\partial v_{j b}}\left(v_{g b}, v_{s b}, v_{d b}\right)=\frac{\partial E_{c}}{\partial v_{j b}}+\frac{\partial E_{g b t}}{\partial v_{j b}}+\frac{\partial E_{t 0}}{\partial v_{j b}} ; j=g, s, d
$$

Though the total $\frac{\partial E}{\partial v}$ is same, the separation of the threshold component makes it possible to derive two simple energy functions, one from the combination of $\frac{\partial E_{c}}{\partial v_{j b}}+\frac{\partial E_{g b t}}{\partial v_{j b}}$, and the other from $\frac{\partial E_{t 0}}{\partial v_{j b}}$. These two energy functions can then be combined to find the total energy function.

\subsubsection{Threshold Energy Function $\left(E_{t 0}\right)$ Calculation}

From (42) and (43), the threshold power can be written as

$$
i_{g 1} v_{t 0}=\frac{\partial E_{t 0}}{\partial v_{g b}} \frac{\partial v_{g b}}{\partial t}+\frac{\partial E_{t 0}}{\partial v_{d b}} \frac{\partial v_{d b}}{\partial t}+\frac{\partial E_{t 0}}{\partial v_{s b}} \frac{\partial v_{s b}}{\partial t}
$$

where $i_{g 1}$ is the gate current. Since $v_{t 0}$ is constant, the threshold energy function can be estimated using

$$
E_{t 0}=\frac{d}{d t}\left(i_{g 1} v_{t 0}\right)=Q_{g} v_{t 0}
$$

where $Q_{g}$ is the gate charge and is given in Table III.

\subsection{2. $E_{c g b t}$ Calculation}

Leaving out the threshold terms, equation (44) reduces to

$$
\frac{\partial E_{c g b t}}{\partial v_{j b}}\left(v_{g b}, v_{s b}, v_{d b}\right)=\frac{\partial E_{c}}{\partial v_{j b}}+\frac{\partial E_{g b t}}{\partial v_{j b}} ; j=g, s, d
$$

which can be solved to find $\frac{\partial E}{\partial v}$, s as

$$
\frac{\partial E_{c g b t}}{\partial v_{g b}}=\frac{1}{2} c_{o x} L\left(2 v_{g b t}-v_{d b}-v_{s b}\right)
$$




$$
\begin{aligned}
& \frac{\partial E_{c g b t}}{\partial v_{s b}}=\frac{1}{2} c_{o x} L\left((1+\alpha) v_{s b}-v_{g b t}\right) \\
& \frac{\partial E_{c g b t}}{\partial v_{d b}}=\frac{1}{2} c_{o x} L\left((1+\alpha) v_{d b}-v_{g b t}\right)
\end{aligned}
$$

The second order partials of equations (48-50) are equal. It shows that an energy function also exist for the sum of remaining gate and channel components. This energy function $E_{c g b t}$ can then be calculated solving the partial differentials with three independent voltage variables $v_{g b}, v_{s b}, v_{d b}$ respectively.

Solving with respect to the gate potential

$$
E_{c g b t}=\int \frac{\partial E_{c g b t}}{\partial v_{g b}} d v_{g b}+E_{1}\left(v_{s b}, v_{d b}\right)=\frac{1}{2} c c_{o x} L\left(v_{g b t 0}{ }^{2}-v_{g b t 0} v_{d b}-v_{g b t 0} v_{s b}\right)+E_{1}\left(v_{s b}, v_{d b}\right)
$$

Solving with respect to the drain potential

$$
E_{c g b t}=\int \frac{\partial E_{c g b t}}{\partial v_{d b}} d v_{d b}+E_{2}\left(v_{g b}, v_{s b}\right)=\frac{1}{4} c_{o x} L(1+\alpha) v_{d b}^{2}-\frac{1}{2} c_{o x} L v_{g b t} v_{d b}+E_{2}\left(v_{g b}, v_{d b}\right)
$$

Solving with respect to the source potential

$$
E_{c g b t}=\int \frac{\partial E_{c g b t}}{\partial v_{s b}} d v_{s b}+E_{3}\left(v_{g b}, v_{d b}\right)=\frac{1}{4} c_{o x} L(1+\alpha) v_{s b}^{2}-\frac{1}{2} c_{o x} L v_{g b t 0} v_{s b}+E_{3}\left(v_{g b}, v_{d b}\right)
$$

Comparing and combining equations for $E_{c g b t}$, the energy function reduces to

$$
E_{c g b t}=\frac{1}{4} c_{o x} L\left(\left(\alpha{\left(v_{d b}\right.}^{2}+v_{s b}^{2}\right)+\left(v_{g b t 0}-v_{d b}\right)^{2}+\left(v_{g b t 0}-v_{s b}\right)^{2}\right)
$$

The total energy function $E$ can now be estimated using (46) and (51) as

$$
E=E_{c g b t}+E_{t 0}
$$

and is shown in Table 3 .

\section{DYNAMIC POWER DISSIPATION MODEL}

The basic idea of dynamic power estimation is that power dissipation comes from trapping the energy stored on a load capacitor by turning off a transistor very quickly. However, it does not make any sense as the first order dynamic power in those cases would be infinite. Also, the energy stored in the switching transistor should be included. In section 4, it was shown that an energy function was possible only from the combination of the gate and the channel conserved power components, which makes it very difficult to know the exact dissipation during the transients using the energy model alone. This is because the conserved components of channel currents are flowing in and out of the source and drain terminals during the transition. When the gate turns off, some of the energy supplied from the gate flows back to the supply through the source terminal while some of the energy gets dissipated from the drain terminal, and there is no way of telling what fraction goes to where without solving for the voltages and currents. 
Charge based dynamic power estimation is an attractive alternate technique. Even though we do not know how much of the stored energy flows out the source and drain, we can always find the total drain charge, $Q_{d}$, and source charge, $Q_{s}$, from Ward's partition [2]. $Q_{s}$ and $Q_{d}$ are not physically separate but act as pseudo-charges, the sum of which constitutes the total channel charge. All they do is give a way to find the currents by taking their time derivatives. Even though there is no physical channel charge partition, it turns out that the current equations act as if they are the time derivatives of the charges. If currents are integrated over a time interval to get the total charges going in or out, it is exactly the same as the changes in $Q_{s}$ and $Q_{d}$. Hence, we can figure out the total charge injected by a transistor without knowing the details of the waveform. All we need to know is the beginning and ending voltage of the transistor terminals and we can figure out $\Delta Q$ for each terminal. This $\Delta Q$ then gets added to the charge on the load capacitor and eventually all gets dissipated.

The total power dissipation is the sum of dynamic power and short circuit power. In contrast with dynamic power, short circuit power cannot be determined without an exact solution to the current and voltage waveforms. Short circuit power comes from the zero order component of the drain current from the turned off transistor. This current component is assumed to be zero in the following derivation.

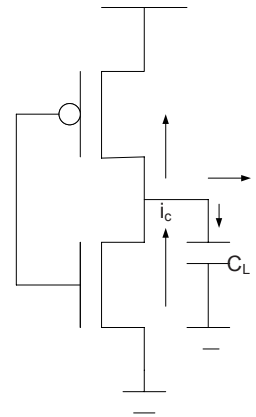

Fig. 3. Charge Based Dynamic Power Model

Fig. 3 shows the charge based dynamic power model. If we define the drain current as positive going in, then the total charge coming out is negative of the integral of the drain current. The zero order turn on current $i_{0}($ on $)$ going from the source to the drain terminal can then be estimated using

$$
-\int i_{0}(\text { on }) d t=-\Delta Q_{D P}-\Delta Q_{D N}-\Delta Q_{L}
$$

where $\Delta Q_{L}$ gives the charge going into the load capacitance. $\Delta Q_{D P}$ and $\Delta Q_{D N}$ are defined as the drain charges at the PFET and the NFET respectively and can be estimated from [11] as

$$
Q_{D P}=-\frac{2}{3} L c_{\text {oxp }}\left(1+\alpha_{p}\right) v d s\left(-\frac{\left(u_{p}-1\right)^{3}}{2 u_{p}-1}+\frac{2}{5} \frac{u_{p}{ }^{5}-\left(u_{p}-1\right)^{5}}{\left(2 u_{p}-1\right)^{2}}\right)
$$




$$
Q_{D N}=-\frac{2}{3} L c_{o x n}\left(1+\alpha_{n}\right) v_{d s}\left(-\frac{\left(u_{n}-1\right)^{3}}{2 u_{n}-1}+\frac{2}{5} \frac{u_{n}^{5}-\left(u_{n}-1\right)^{5}}{\left(2 u_{n}-1\right)^{2}}\right)
$$

where $u_{p}=\frac{v_{g s}-v_{t p}}{\left(1+\alpha_{p}\right) v_{d s}}, u_{n}=\frac{v_{g s}-v_{t n}}{\left(1+\alpha_{n}\right) v_{d s}}, c_{\text {oxp }}$ and $c_{\text {oxn }}$ are the oxide capacitance per unit lengths, $\alpha_{p}$ and $\alpha_{n}$ are the bulk charge coefficients and $v_{t p}$ and $v_{t n}$ are threshold voltages of PFET and NFET respectively. The change in charge at the ground or the supply can then be estimated using

$$
\Delta Q=-\int_{0}(\text { on }) d t-\Delta Q_{S}-\Delta Q_{B}
$$

where $\Delta Q_{S}$ and $\Delta Q_{B}$ are defined as the changes in source and the substrate charges. Using this approach, the difference in charge at ground due to a falling transient can be estimate using

$$
\Delta Q_{f}=-\Delta Q_{D P f}-\Delta Q_{D N f}-\Delta Q_{L f}-\Delta Q_{S N f}-\Delta Q_{B N f}
$$

and the difference in charge at ground due to the rising transient becomes

$$
\Delta Q_{r}=-\Delta Q_{S N r}-\Delta Q_{B N r}=\Delta Q_{S N f}+\Delta Q_{B N f}
$$

which is correct if the short circuit power is zero. For a rising transient, as mentioned above, zero order components are assumed to be zero. Hence we only get the reverse changes in $\Delta Q_{S}$ and $\Delta Q_{B}$. The difference in charge $\Delta Q$ can then be rewritten as

$$
\Delta Q=\Delta Q_{r}+\Delta Q_{f}=-\Delta Q_{D P f}-\Delta Q_{D N f}-\Delta Q_{L f}
$$

In equation (58), the last term represents the normal component present in the conventional dynamic power model, while our model adds two extra terms responsible for the first order power dissipation from charge stored in the channel. The dynamic power dissipation is then given by

$$
P=\Delta Q V_{d d} f=\left(\Delta Q_{r}(\text { final })-\Delta Q_{r}(\text { initial })\right)+\left(\Delta Q_{f}(\text { final })-\Delta Q_{f}(\text { initial })\right) V_{d d} f
$$

which can be solved for a falling transient as

\section{For the PFET:}

$$
\begin{aligned}
& Q_{D P}(\text { initial })=\left.Q_{D P}\right|_{V} \rightarrow 0 \\
& Q_{D P}(\text { final })=\left.Q_{D P}\right|_{V} \rightarrow-V_{d d}, V_{d s} \rightarrow 0 \\
& =\frac{1}{2} c_{o x p}\left(V_{d d}+V_{t p}\right)
\end{aligned}
$$

From (60) and (61)

$$
\Delta Q_{D P}=\frac{1}{2} c_{\text {oxp }}\left(V_{d d}+V_{t p}\right)
$$

For the NFET:

$$
Q_{D N}(\text { initial })=\left.Q_{D N}\right|_{V S} \rightarrow V_{d d}, V_{d s} \rightarrow 0=-\frac{1}{2} c{ }_{\text {oxn }}\left(V_{d d}-V_{t n}\right)
$$




$$
Q_{D N}(\text { final })=\left.Q_{D N}\right|_{V_{g S} \rightarrow 0}=0
$$

From (63) and (64)

$$
\Delta Q_{D N}=\frac{1}{2} c_{\text {oxn }}\left(V_{d d}-V_{t n}\right)
$$

For the load:

$$
\begin{aligned}
& Q_{L}(\text { initial })=\left.C_{L} V_{d b}\right|_{V} \rightarrow 0 \\
& Q_{L}(\text { final })=\left.C_{L} V_{d b}\right|_{V_{d b}} \rightarrow v_{d d}=C_{L} V_{d d}
\end{aligned}
$$

From (66) and (67)

$$
\Delta Q_{L}=C_{L}{ } d d
$$

From (62), (65) and (68)

$$
\Delta Q=C_{L} V_{d d}+\frac{1}{2} c_{\text {oxn }}\left(V_{d d}-V_{t n}\right)+\frac{1}{2} c_{\text {oxp }}\left(V_{d d}+V_{t p}\right)
$$

Substituting $\Delta Q$ in (59), the dynamic power equation reduces to

$$
P=C_{L} V_{d d}{ }^{2} f+\left(\frac{1}{2} c_{\text {oxn }}\left(V_{d d}-V_{t n}\right) V_{d d}+\frac{1}{2} c_{\text {oxp }}\left(V_{d d}+V_{t p}\right) V_{d d}\right) f
$$

Equation (70) shows the presence of extra dynamic power component to the conventional dynamic power equation.

\section{FIRST ORDER CURRENT COMPONENTS}

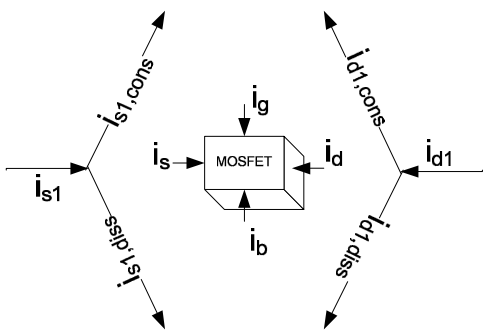

Fig. 4: First order dissipative and conserved current components

As seen from Table I, first order current is a function of terminal voltages and their time derivatives, and as mentioned above, the coefficient of $d v / d t$ instead of representing purely storage capacitance, is also responsible for some of the power dissipation in the channel. This suggests that the first order drain and the source currents consist of two separate components, 
one that contributes to power dissipation in the channel, and another that is responsible for the energy storage. Taking this approach, $i_{d l}$ and $i_{s l}$ can be expanded as

$$
\begin{aligned}
& i_{d 1}=i_{d 1, \text { cons }}+i_{d 1, \text { diss }} \\
& i_{s 1}=i_{s 1, \text { cons }}+i_{s 1, \text { diss }}
\end{aligned}
$$

Fig. 4 shows this concept where first order currents $i_{d l}$ and $i_{s l}$ are separated into two components. Since the gate and the substrate currents are non-dissipative in the absence of leakage, there is no need to separate them.

The dissipative current components in equations (71) and (72) are due to the first order power dissipation in the channel from the charge redistribution and is computed by dividing the power dissipated in the channel by the drain to source potential

$$
i_{d 1, \text { diss }}=\frac{P_{c 1, \text { diss }}}{{ }^{d} d s}=i_{t t, \text { diss }}=-i{ }_{s 1, \text { diss }}
$$

$i_{t t, \text { diss }}$ in equation (73) is the trans-capacitive transport current that is responsible for the extra power dissipation in the channel, and is defined as positive going into the drain. The conserved drift component can now be computed by subtracting the dissipated component from the total first order current.

$$
\begin{aligned}
& i_{d 1, \text { cons }}=i_{d 1}-i_{t t, \text { diss }} \\
& i_{s 1, \text { cons }}=i_{s 1}+i_{t t, \text { diss }}
\end{aligned}
$$

Separation of currents into conserved and dissipative terms helps to compute the true energy conserving capacitances. True in the sense that these capacitances are estimated simply from the conserved components of current using equations (74) and (75).

$$
i_{i 1, \mathrm{cons}}=C_{c i i} \partial_{t} v_{i b}-\sum_{j \neq i, b}\left(C_{c i j} \partial_{t} v_{j b}\right) ; i, j=g, d, s, b .
$$

where $C_{c i i}, C_{c i j}$ are the conserved components of the capacitor. In equation (76) and all the subsequent equations, the subscript notation ' $c$ ' or ' $d$ ' is used for conserved or dissipative components. Table 4 and 5 summarize the conserved and dissipative components of currents and capacitances.

\section{EQUIVALENT CIRCUIT}

In this section, we develop an equivalent circuit by following the method used by LimFossum [11]. Table IV showed that the capacitances were not reciprocal, which makes the capacitance representation using two terminal reciprocal capacitances impossible if these capacitances are made to represent the total first order drain current. However, equation (74) can be rewritten with reciprocal capacitors as

$$
i_{d 1, \text { cons }}=C_{g d} \partial_{t} v_{d g}+C_{b d} \partial_{t} v_{d b}+i_{t t, \text { cons }}
$$

where

$$
i_{t t, \text { cons }}=\left(C_{g d}-C_{c d g}\right) \partial_{t} v_{g b}+\left(C_{c s d}-C_{c d s}\right) \partial_{t} v_{s b}+C_{c s d} \partial_{t} v_{d s}
$$


The dissipative component of current from equation (73) can also be written in terms of dissipative capacitances as

$$
{ }^{i} d 1, \text { diss }=C_{d d d} \partial^{\partial} v^{d} d b-C_{d d g}{ }^{\partial} t
$$

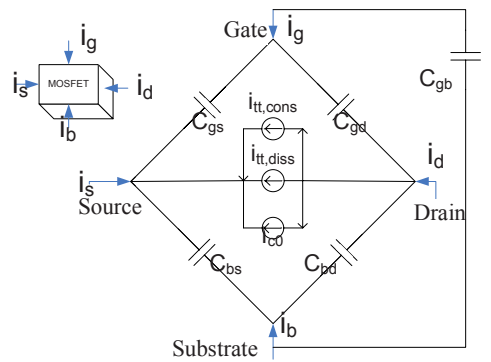

Fig. 5: Equivalent Circuit for a four terminal SOI MOSFET

Fig. 5 shows an equivalent circuit of a four terminal MOSFET. The model is equivalent to Lim-Fossum [11], but we have separated the trans-capacitive transport current, $i_{t t}$ into conserved and dissipative components. There are three current components flowing from the drain to the source terminal. The current component responsible for the first order power dissipation in the channel is represented by $i_{t, d i s s}$ the conserved current component is represented by $i_{t t, c o n s} . I_{c 0}$ represents the steady state zero order current. The two terminal reciprocal capacitances $C_{g d}, C_{g s}, C_{b d}, C_{b s}$ and $C_{g b}$ represents the conserved gate to drain, gate to source, substrate to source, substrate to drain and gate to substrate capacitances respectively. The two terminal capacitances do not conserve energy by themselves; the conserved component of $i_{t t}$ must be included. $C_{d d d}, C_{d d g}, C_{d d s}$ in equation (79) represents the dissipative drain to drain, drain to gate and drain to source capacitances respectively.

\section{MODEL VALIDATION AND COMPARISON}

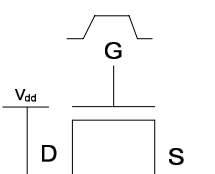

(a)

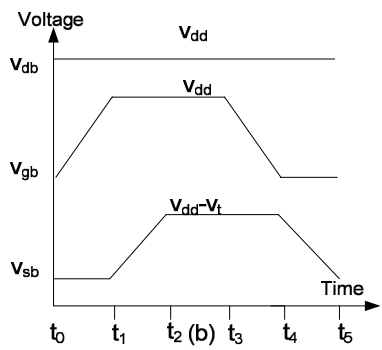

Fig. 6: (a) Pass transistor logic (b) Voltage Waveforms

In this section, we have shown that the gate is leakage free and does not contribute any net charge to the channel. We have also validated the fact that our first order power dissipation model is indeed a charge conserving, as the total charge over a complete cycle is conserved. 
Fig. 6 shows the idealized voltage waveforms for the drain, gate and the source terminals used to pass the logic through the NFET. The drain terminal is assumed to be high during the entire simulation, while the gate and the source potentials goes through many transitions. In the first transition $\left(t_{0}\right.$ to $\left.t_{1}\right)$, the gate terminal goes from low at $t_{0}$ to high at $t_{l}$, while the source potential remains low. The transistor enters the saturation as soon as the gate to source potential becomes greater than the threshold voltage $v_{t}$. The extra gate charge $\Delta Q_{g 1}$, is then given by

$$
\begin{aligned}
\Delta Q_{g 1} & =\left(c_{g g} \Delta v_{g b}\right)_{\text {cutoff }}+\left(c_{g g} \Delta v_{g b}\right)_{\text {saturation }} \\
& =\frac{\alpha}{1+\alpha} c_{o x} v_{t 0}+\left(\frac{1}{3} \frac{\alpha}{1+\alpha}+\frac{2}{3}\right) c_{o x}\left(v_{d d}-v_{t 0}\right)
\end{aligned}
$$

During the second transition ( $t_{1}$ to $\left.t_{2}\right)$, the gate terminal stays high $\left(v_{d d}\right)$ and the pass transistor remains in the saturation. The source terminal on the other hand, goes from low (0) to high $\left(\frac{v d d-v}{1+\alpha}\right)$ and the extra gate charge $\Delta Q_{g 2}$ becomes

$$
\Delta Q_{g 2}=-\left(c_{g s} \Delta v_{s b}\right)_{\text {saturation }}=-\frac{2}{3} c c_{o x} \frac{v_{d d}-v_{t 0}}{1+\alpha}
$$

The transistor now enters the cutoff (at $t_{2}$ ) and remains there even though the gate and source terminals come back to its original states at $t_{4}$ and $t_{5}$. The extra gate charge during these transitions are given by

$$
\Delta Q_{g 3}=\left(c_{g g}^{\Delta v_{g b}}\right)_{\text {cutoff }}=-\frac{\alpha}{1+\alpha} c x^{v} d d
$$

The total gate charge $\Delta Q_{g}$ is then calculated by adding the extra gate contributions as

$$
\Delta Q_{g}=\sum_{i=1}^{3} \Delta Q_{g i}=0
$$

For a complete cycle, charge is conserved and there is no extra non-zero contribution from the gate. Fig. 7 shows this concept using a two dimensional profile, where $v_{s b}=v_{g b t 0} /(1+\alpha)$ sets a boundary between the cutoff and saturation regions. Simulation is started at some point $\mathrm{A}$, and goes through transitions $\mathrm{B}, \mathrm{C}$ and $\mathrm{D}$, before settling back to its initial state at $\mathrm{A}$.

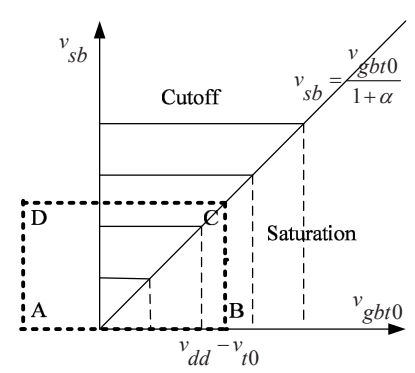

Fig. 7 : Charge Profile

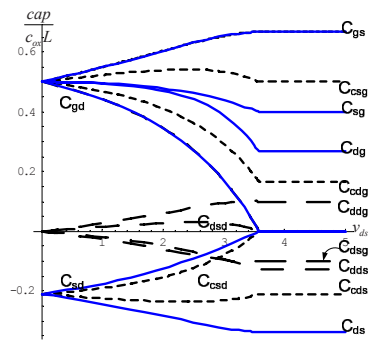

Fig. 8: Capacitance Plots vs. $\mathrm{v}_{\mathrm{ds}}$ 
Our model also verifies that Ward's method of charge partitioning works correctly as long as the body charge has a linear dependence on the channel potential. It predicts the same source and the drain currents, and hence the same terminal capacitances. However, we are able to partition these capacitances into conserved and dissipative components, as shown in Fig. 8. The partitioning approach to capacitances offers several advantages over conventional transcapacitances.

- The energy stored in the conserved capacitances can be predicted.

- They can be made to agree with Meyer's capacitances [1] if the body effect and body bias are ignored.

Our other significant contribution has been in the power estimation. Our models have improved the device power measurements by implementing two important concepts:

- First order terms have to be included for power dissipation estimation as they become significant at higher frequencies.

- Stored components can be ignored for computationally efficient power dissipation estimation.

The average device power, $\bar{P}$, is then possible by taking dissipative current times voltage and integrating them over time. A simple simulation can be used to show the importance of first order power.

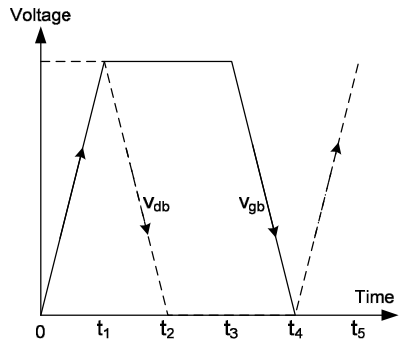

Fig. 9: $v_{\mathrm{gb}}$ and $\mathrm{v}_{\mathrm{db}}$ waveforms

Fig. 9 shows the idealized voltage waveforms for the drain and the gate terminals used for simulation of turning a transistor on then off. The average first order dissipative power from the first transition $\left(v_{d s}=v_{d d}\right)$ when $v_{g b}$ goes from low at $t_{0}$ to high at $t_{l}$ is computed by

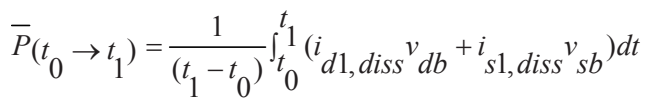

If we assume the source and the substrate are at the same potential $\left(v_{s b}=0\right)$, equation (84) can be rewritten as

$$
\bar{P}\left(t_{0} \rightarrow t_{1}\right)=\frac{1}{\left(t_{1}-t_{0}\right)} \int_{t_{0}}^{t}\left(i^{1} d 1, \text { diss }^{v} d b\right) d t
$$

In the second power dissipating transition, when the gate terminal is high, the drain swings from high at $t_{1}$ to low at $t_{2}$. The dissipative power equation (84) reduces to

$$
\bar{P}\left(t_{1} \rightarrow t_{2}\right)=\frac{1}{\left(t_{2}-t_{2}\right)} \int_{t_{1}}^{t_{2}} i_{d 1, \operatorname{diss}^{v} d b} d t
$$


During the interval $t_{2}$ to $t_{4}$, there is no power dissipation in the channel $\left(v_{d s}=0\right)$. The final power transition occurs when the drain waveform swings from low at $t_{4}$ to high at $t_{5}$. As the gate voltage has already reached a steady low value, the power equation becomes

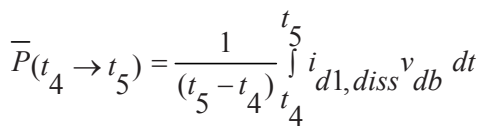

The total dissipative power for a complete cycle is computed taking the sum of all these powers as

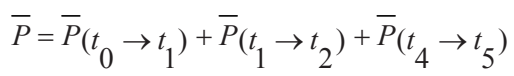

For a complete cycle, energy is conserved. This allows us to leave out the conserved component from the power equation for computationally efficient power dissipation predictions [13]. Nonetheless, the total dissipative power includes the first order terms as predicted by equation (88). These first order dissipative components become significant at higher frequencies and modify the total power dissipated in the channel as shown in Fig. 10. The total power is no longer constant, and at high frequencies becomes dependent on the switching frequencies.
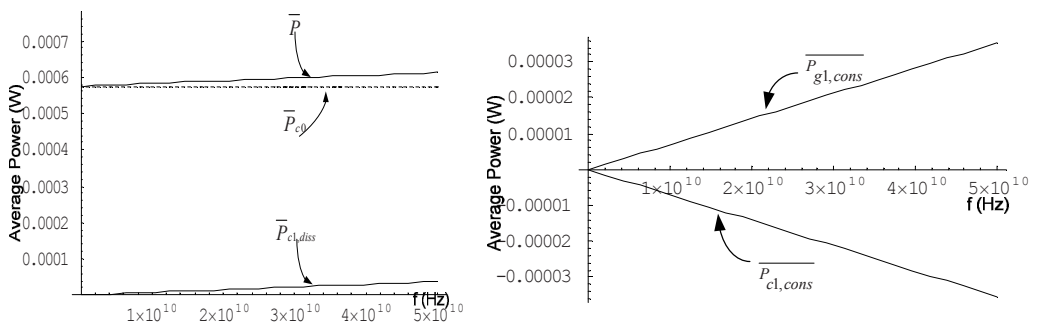

Fig. 10: Dissipative Power vs. Frequency Fig. 11: Conserved Power vs. Frequency

The result also shows that we need to be extra careful while doing the power measurements. It is not appropriate to look only at the channel dissipation; the first order power dissipation does have contributions from the gate. If the power dissipation is estimated by just considering the total channel power, there would be an extra negative component from the conserved energy. In that case, the channel could act as an energy generator. In reality, that is not the case. Power is pumped from the gate to the channel and when the contribution from the gate is added, the conserved terms cancel out (Fig. 11).

\section{CONCLUSIONS}

The development of a self consistent, quasi-static, first order power dissipation model for a fully depleted SOI MOSFET has been described. The Lim-Fossum current and charge model has been verified as correct to first order even though the Ward partition of source and drain charge was used. The transient current is separated into conserved and dissipative components. Significance of higher order power dissipation at higher frequencies is discussed. The existence of energy function also is validated to make the power dissipation estimation computationally efficient. 


\section{REFERENCES}

[1] J.E. Meyer, "MOS models and circuit simulation," RCA Rev., vol. 32, pp. 42-63, 1971.

[2] D.E.Ward, "Charge-Based Modeling of Capacitance in MOS Transistors", Stanford Electronics Lab., Stanford University, Tech. Rep. F201-11, June 1981.

[3] B. J. Sheu, D. L. Scharfetter, C. Hu and D. O. Pederson, "A compact IGFET charge model”, IEEE Transactions on Circuits Systems, vol. CAS-31, pp 745-748, 1984.

[4] K.A. Sakallah, Yao-Tsung Yen, and S.S.Greenberg, "A first-order charge conserving MOS capacitance model," IEEE Transactions on Computer-Aided Design, vol. 9, pp. 99-108, January 1990.

[5] S. S. Chung, "A charge-based capacitance model of short-channel MOSFET's," IEEE Transactions on Computer-Aided Design, vol. 8, no. 1, January 1989.

[6] M. A. Cirit, "The Meyer model revisited: why is charge not conserved?" IEEE Transactions on Computer- Aided Design, vol. CAD-8, pp. 1033-1037, October 1989.

[7] P. Yang, B.D. Epler and P. K. Chatterjee, "An Investigation of the Charge Conservation Problem for MOSFET Circuit Simulation", IEEE Journal of Solid-State Circuits, Vol. SC-18, February 1983.

[8] A. S. Roy, C. C. Enz and Jean-Michel Sallese, "Source-Drain Partitioning in MOSFET", IEEE Trans. On Elec. Devices, vol. 54, No. 6, June 2007.

[9] A. Aarts, R. van der Hout, J. Paasschens, A. Scholten, M. Willemsen, and D. Klaassen, "New fundamental insight into capacitance modeling of laterally nonunion MOS devices, "IEEE Trans. Electron Devices, vol. 53, No. 2, pp. 270-278, Feb 2006.

[10] J.G. Fossum, H. Jeong, and S. Veeraraghavan, "Significance of the channel-charge partition in the transient MOSFET model," IEEE Transactions on Electron Devices, vol. Ed-33, no.10, October 1986.

[11] H. Lim and J. Fossum, "A charge-based large-signal model for thin-film SOI MOSFET's", IEEE Journal of Solid-State Circuits, Vol. Sc-20, no.1, February 1985.

[12] W. Lie, M. Chang, "Transistor Transient Studies Including Trans-Capacitive Current and Distributive Gate Resistance for Inverter Circuits", IEEE Trans. on Circuits and Systems, Vol. 45, April 1998.

[13] S. Sharma, "First Order, Quasi-Static, Charge Conserving MOSFET Channel Capacitance Model," PhD. Dissertation, Oklahoma State University, December 2007.

[14] S. Y. Oh, D. E. Ward and R. W. Dutton, "Transient analysis of MOS transistors", IEEE J. Solid-State Circuits, vol. SC-15, pp. 636-643, 1980.

[15] Y.Cheng and C. Hu, "MOSFET modeling and BSIM3 user's guide", Kluwer Academic Publishers, 1999.

[16] William Liu, "MOSFET models for SPICE simulation, including BSIM3v4 and BSIM4", John Wiley and Sons, Inc., 2001.

[17] Yannis Tsividis, "Operation and the Modeling of The MOS Transistors", Oxford University Press, June 2003.

[18] Wolfram Reseach, Inc., Mathematica, Version 5.2, Champaign, IL (2005). 
Table 1: Charge and Current Equations for NMOS

\begin{tabular}{|c|c|c|c|}
\hline & Linear & Saturation & Cut-Off \\
\hline & $\begin{array}{l}q_{c}<0 \\
v_{g d t}>0 \\
v_{g s t}>0\end{array}$ & $\begin{array}{l}q_{c}<0 \\
v_{g d t}=0 \\
v_{g s t}>0\end{array}$ & $\begin{array}{l}q_{c}=0 \\
v_{g d t}=0 \\
v_{g s t}=0\end{array}$ \\
\hline$q_{s}$ & $-c_{o x} v_{g s t}$ & $-c_{\text {ox }} v_{\text {gst }}$ & 0 \\
\hline$q_{d}$ & $-c_{o x}{ }^{v} g d t$ & 0 & 0 \\
\hline$I_{c 0}$ & $\frac{\mu c}{2 L(1+\alpha)}\left(v_{g s t}^{2}-v_{g d t}^{2}\right)$ & $\frac{\mu c}{2 L(1+\alpha)} v_{g s t}^{2}$ & 0 \\
\hline$i_{s 1}$ & $\frac{2 c_{o x} L}{15\left(v_{g d t}+v_{g s t}\right)^{3}}\left[2 v_{g d t}\left(\frac{d}{d t} v_{g d t}\right)\left(v_{g d t}^{2}+3 v_{g d t} v_{g s t}+v_{g s t}^{2}\right)+v_{g s t}\left(\frac{d}{d t} v_{g s t}\right)\left(8 v_{g d t}^{2}+9 v_{g d t} v_{g s t}+3 v_{g s t}^{2}\right)\right]$ & $\frac{2}{5} c_{o x} L \frac{d}{d t} v_{g s t}$ & 0 \\
\hline$i_{d 1}$ & $\frac{2 c_{o x} L}{15\left(v_{g d t}+v_{g s t}\right)^{3}}\left(v_{g d t}\left(\frac{d}{d t} v_{\text {gdt }}\right)\left(3 v_{g d t}^{2}+9 v_{g d t} v_{g s t}+8 v_{g s t}^{2}\right)+2 v_{g s t}\left(\frac{d}{d t} v_{g s t}\right)\left(v_{g d t}^{2}+3 v_{g d t} v_{g s t}+v_{g s t}^{2}\right)\right)$ & $\frac{4}{15} c c_{o x}^{L} \frac{d}{d t} v_{g s t}$ & 0 \\
\hline
\end{tabular}

Table 2: Power Equations

\begin{tabular}{|c|c|c|c|}
\hline & Linear & Saturation & Cut-Off \\
\hline$P_{c 0}$ & $\frac{\mu c_{o x}}{2 L(1+\alpha)} v_{d s}\left(v_{g s t}^{2}-v_{g d t}^{2}\right)$ & $\frac{\mu c_{o x} v_{d s} v_{g s t}^{2}}{2 L(1+\alpha)}$ & 0 \\
\hline$P_{c 1, \text { diss }}$ & $c_{o x} L \frac{v_{d s}\left(v_{g s t}-v_{g d t}\right)}{30\left(v_{g d t}+v_{g s t}\right)^{3}}\left(3 v_{g d t}^{2} \frac{d}{d t} v_{g d t}+3 v_{g s t}^{2} \frac{d}{d t} v_{g s t}+7 v_{g d t}\left(\frac{d}{d t} v_{g d t}+\frac{d}{d t} v_{g s t}\right) v_{g s t}\right)$ & $\frac{c_{o x} L v_{d s}}{10} \frac{d v_{g s t}}{d t}$ & 0 \\
\hline$P_{c 1, \text { cons }}$ & $\frac{-c x}{6(1+\alpha)}\left(-3\left(v_{g d t} \frac{d}{d t} v_{g d t}+v_{g s t} \frac{d}{d t} v_{g s t}\right)+\frac{4\left(v_{g d t} \frac{d}{d t} v_{g d t}\left(v_{g d t}+2 v_{g s t}\right)+v_{g s t} \frac{d}{d t} v_{g s t}\left(2 v_{g d t}+v_{g s t}\right) v_{g b t 0}\right)}{\left(v_{g d t}+v_{g s t}\right)^{2}}\right.$ & $\begin{array}{l}-\frac{c o x}{6(1+\alpha)} \frac{d}{d t} v_{g s t} \\
\left(4 v_{g b t 0}-3 v_{g s t}\right)\end{array}$ & 0 \\
\hline
\end{tabular}

Table 3: Energy Function

\begin{tabular}{||c|c|c|c||}
\hline \hline$Q_{g}$ & Linear & Saturation & Cut-Off \\
\hline$c_{o x} L\left(v_{g b}-v_{f b}-\phi-v_{s b}-\frac{v_{d b}-v_{s b}}{2}+\frac{(1+\alpha)\left(v_{d b}-v_{s b}\right)^{2}}{12\left(v_{g s t}-(1+\alpha)\left(v_{d b}-v_{s b}\right) / 2\right)}\right)$ & $c_{\alpha x} L\left(v_{g b}-v_{f b}-\phi-v_{s b}-\frac{v_{g s t}}{3(1+\alpha)}\right)$ & $\left.\begin{array}{l}c_{o x} L\left(v_{g b}\right. \\
-v_{f b}-\phi \\
v_{g b t 0}\end{array}\right)$ \\
\hline$E_{f}$ & $\frac{1}{4} c_{o x} L\left(\alpha\left(v_{d b}^{2}+v_{s b}^{2}\right)+\left(v_{d b}-v_{g b t 0}\right)^{2}+\left(v_{g b t 0}-v_{s b}\right)^{2}\right)+Q_{g} v_{t 0}$ & $\frac{1}{4} c_{o x} L\left(\alpha\left(\frac{v_{g b t 0}}{1+\alpha}+v_{s b}^{2}\right)\right.$ & $\frac{c_{o x} \alpha L}{2} \frac{v_{g b t 0}^{2}}{1+\alpha}$ \\
$\left.+\left(v_{g b t 0}-v_{s b}\right)^{2}\right)+Q_{g} v_{t 0}$ \\
$+Q_{g} v_{t 0}$
\end{tabular}


FIRST ORDER, QUASI-STATIC, SOI CHARGE CONSERVING POWER

DISSIPATION MODEL 309

Table 4: Conserved and Dissipative Current Components

\begin{tabular}{|c|c|c|c|}
\hline & Linear & Saturation & Cut-Off \\
\hline $\begin{array}{l}i_{t t, d i s s}= \\
-i_{s 1, d i s s}\end{array}$ & $\frac{c_{o x} L\left(v_{g s t}-v_{g d t}\right)}{30\left(v_{g d t}+v_{g s t}\right)^{3}}\left(3\left(v_{g d t}^{2} \frac{d}{d t} v_{g d t}+v_{g s t}^{2} \frac{d}{d t} v_{g s t}\right)+7 v_{g d t} v_{g s t}\left(\frac{d}{d t} v_{g d t}+\frac{d}{d t} v_{g s t}\right)\right)$ & $\frac{c_{o x} L}{10} \frac{d}{d t} v_{g s t}$ & 0 \\
\hline$i_{d 1, \text { cons }}$ & $\frac{c_{o x} L}{6\left(v_{g d t}+v_{g s t}\right)^{2}}\left(v_{g d t} \frac{d}{d t} v_{g d t}\left(3 v_{g d t}+5 v_{g s t}\right)+v_{g s t} \frac{d}{d t} v_{g s t}\left(3 v_{g d t}+v_{g s t}\right)\right)$ & $\frac{c_{o x}{ }^{L}}{6} \frac{d}{d t} v_{g s t}$ & 0 \\
\hline$i_{s 1, \text { cons }}$ & $\frac{c_{o x} L}{6\left(v_{g d t}+v_{g s t}\right)^{2}}\left(v_{g d t} \frac{d}{d t} v_{g d t}\left(v_{g d t}+3 v_{g s t}\right)+v_{g s t} \frac{d}{d t} v_{g s t}\left(5 v_{g d t}+3 v_{g s t}\right)\right)$ & $\frac{c_{o x}{ }^{L}}{2} \frac{d}{d t} v_{g s t}$ & 0 \\
\hline
\end{tabular}

Table 5: Conserved and Dissipative Capacitances

\begin{tabular}{|c|c|c|c|}
\hline & Linear & Saturation & Cut-Off \\
\hline$C_{g b}$ & $\frac{\alpha}{3(1+\alpha)} c_{o x} L \frac{\left(v_{g d t}-v_{g s t}\right)^{2}}{\left(v_{g d t}+v_{g s t}\right)^{2}}$ & $\frac{\alpha}{3(1+\alpha)} c_{o x} L$ & $\frac{\alpha c_{o x} L}{(1+\alpha)}$ \\
\hline$C_{g d}$ & $\frac{2}{3} c_{o x} L v_{g d t} \frac{\left(v_{g d t}+2 v_{g s t}\right)}{\left(v_{g d t}+v_{g s t}\right)^{2}}$ & 0 & 0 \\
\hline$C_{g s}$ & $\frac{2}{3} c_{o x} L v_{g s t} \frac{\left(2 v_{g d t}+v_{g s t}\right)}{\left(v_{g d t}+v_{g s t}\right)^{2}}$ & $\frac{2}{3} c_{o x} L$ & 0 \\
\hline$C_{c s g}$ & $\frac{1}{6} c_{o x} L \frac{\left(v_{g d t}^{2}+8 v_{g d t} v_{g s t}+3 v_{g s t}^{2}\right)}{\left(v_{g d t}+v_{g s t}\right)^{2}}$ & $\frac{1}{2} c_{o x}^{L}$ & 0 \\
\hline$C_{c s b}$ & $\alpha C_{c s g}$ & $\alpha C_{c s g}$ & 0 \\
\hline$C_{c s d}$ & $-\frac{(1+\alpha)}{6} c_{o x} L \frac{v_{g d t}\left(v_{g d t}+3 v_{g s t}\right)}{\left(v_{g d t}+v_{g s t}\right)^{2}}$ & 0 & 0 \\
\hline$C_{c d g}$ & $\frac{1}{6} c_{o x} L \frac{\left(3 v_{g d t}{ }^{2}+8 v_{g d t} v_{g s t}+v_{g s t}{ }^{2}\right)}{\left(v_{g d t}+v_{g s t}\right)^{2}}$ & $\frac{1}{6} c_{o x} L$ & 0 \\
\hline$C_{c d b}$ & $\alpha C_{c d g}$ & $\alpha C_{c d g}$ & 0 \\
\hline$C_{c d s}$ & $-\frac{(1+\alpha)}{6} c_{o x} L \frac{v_{g s t}\left(3 v_{g d t}+v_{g s t}\right)}{\left(v_{g d t}+v_{g s t}\right)^{2}}$ & $-\frac{1+\alpha}{6} c_{o x} L$ & 0 \\
\hline$C_{d d g}$ & $c_{o x} L\left(v_{g s t}-v_{g d t}\right)\left(3 v_{g d t}^{2}+14 v_{g d t} v_{g s t}+3 v_{g s t}^{2}\right) / 30\left(v_{g d t}+v_{g s t}\right)^{3}$ & $\frac{1}{10} c_{o x} L$ & 0 \\
\hline$C_{d d s}$ & $-(1+\alpha) c_{o x} L\left(v_{g s t}-v_{g d t}\right) v_{g s t}\left(7 v_{g d t}+3 v_{g s t}\right) / 30\left(v_{g d t}+v_{g s t}\right)^{3}$ & $-\frac{1+\alpha}{10} c_{o x} L$ & 0 \\
\hline$C_{d d b}$ & $-\alpha C_{d d g}$ & $-\frac{\alpha}{10} c_{o x} L$ & 0 \\
\hline$C_{d s g}$ & $-C_{d d g}$ & $-C_{d d g}$ & 0 \\
\hline$C_{d s d}$ & 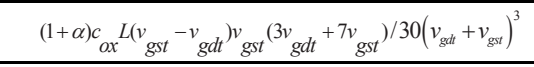 & 0 & 0 \\
\hline$C_{d s b}$ & $-C_{d d b}$ & $-C_{d d b}$ & 0 \\
\hline
\end{tabular}

